\title{
Utilizing 5-aminolevulinic acid and pulsed dye laser for photodynamic therapy of oral leukoplakia - pilot study
}

\author{
Gal Shafirstein ${ }^{1 *}$, Wolfgang Bäumler ${ }^{2}$, Eric Sigel ${ }^{3}$, Chun-Yang Fan ${ }^{4,5}$, Kimberly Berry ${ }^{1}$, Emre Vural ${ }^{1,5}$, \\ Brendan C Stack', James Y Suen ${ }^{1}$ \\ From 2nd Scientific Meeting of the Head and Neck Optical Diagnostics Society \\ San Francisco, CA, USA. 23-24 January 2010
}

\section{Objective}

Determine the safety and efficacy of photodynamic therapy (PDT) in the treatment of oral leukoplakia with 5-aminolevulinic acid (5-ALA) and pulsed dye laser (PDL).

\section{Methods}

A total of 24 subjects, between 18 - 80 years old, with a confirmed diagnosis of leukoplakia with or without dysplasia, measuring at least $10 \mathrm{~mm}$ in diameter were enrolled in cohorts of 3 within 24 months. Twenty percent solution of 5-ALA, Levulan Kerastick ${ }^{\mathrm{TM}}$ were applied to the lesions, for 1 to 3 hours, by utilizing custom fixtures made from hygienic polymer. The drug application was confirmed with Fluorescence diagnosis system $\left(\right.$ Dyaderm $\left.^{\mathrm{TM}}\right)$. High power pulsed dye laser emitting $585-\mathrm{nm}$ wavelength was used to activate the 5-ALA. The laser dose was escalated from 6 to $8 \mathrm{~J} / \mathrm{cm}^{2}$. The safety was evaluated via clinical observation 48 hours post treatment, and the clinical and pathological efficacy were evaluated 30 and days after treatment, respectively. Histopathology and immunohistochemistry were conducted on fixed tissue samples collected at screening and 90 days post treatments, from the lesion and an adjacent healthy site.

\section{Results}

No significant adverse events and minor pain (3 out of 10) was observed, during and following PDT in the safety phase of the study. The maximum tolerated dose was $8 \mathrm{~J} / \mathrm{cm}^{2}$. Partial response was observed in $54 \%$ of the treated subjects and significant response was observed in $46 \%$ of the subject treated in the efficacy phase of the study.

\section{Conclusions}

Photodynamic therapy (PDT) with 5-Aminolevulinic Acid (5-ALA) and PDL could be use to regress oral leukoplakia. The treatment is safe and well tolerated. Thick and progress lesions require multiple treatments. An application time of 2 hours and laser radiant exposure of $8 \mathrm{~J} / \mathrm{cm}^{2}$ were found to be the best settings, in this study. The high power laser allows completing the laser therapy within 1-3 minutes. More work is underway to determine the optimal laser radiant exposure and drug application to improve the rate of complete regression.

\section{Author details}

'Department of Otolaryngology, University of Arkansas for Medical Sciences, Little Rock, United States. '2Department of Dermatology, Regensburg University, Regensburg, Germany . ${ }^{3}$ Department of Biostatistics, University of Arkansas for Medical Sciences, Little Rock, USA . ${ }^{4}$ Department of Pathology, University of Arkansas for Medical Sciences, Little Rock, USA . ${ }^{5}$ Medical Research Service, Central Arkansas Veterans Healthcare System, Little Rock, USA.

Published: 29 October 2010 